This is the accepted manuscript version of the paper published in: Marchesi S., Roselli C., Wykowska A. (2021) Cultural values, but not nationality, predict social inclusion of robots. In $13^{\text {th }}$ Internal

Conference on Social Robotics. Singapore.

Lecture Notes in Computer Science, 13086, 48-57.

https://doi.org/10.1007/978-3-030-90525-5_5

\title{
Cultural values, but not nationality, predict social inclusion of robots
}

\author{
Serena Marchesi* 1, 2 [0000-0001-9931-156X], Cecilia Roselli* 1, 3 [0000-0002-0657-8108], and \\ Agnieszka Wykowska 1 [0000-0003-3323-7357] \\ ${ }^{1}$ Social Cognition in Human Robot Interaction, Italian Institute of Technology, Genova, 16152 Italy \\ ${ }^{2}$ Department of Computer Science, Faculty of Science and Engineering, Manchester University, Manches- \\ ter, United Kingdom \\ ${ }^{3}$ DIBRIS, Dipartimento di Informatica, Bioingegneria, Robotica ed Ingegneria dei Sistemi, Genova, 16145, \\ Italy \\ Agnieszka.Wykowska@iit.it
}

\begin{abstract}
Research highlighted that Western and Eastern cultures differ in socio-cognitive mechanisms, such as social inclusion. Interestingly, social inclusion is a phenomenon that might transfer from human-human to human-robot relationships. Although the literature has shown that individual attitudes towards robots are shaped by cultural background, little research has investigated the role of cultural differences in the social inclusion of robots. In the present experiment, we investigated how cultural differences, in terms of nationality and individual cultural stance, influence social inclusion of the humanoid robot $\mathrm{iCub}$, in a modified version of the Cyberball game, a classical experimental paradigm measuring social ostracism and exclusion mechanisms. Moreover, we investigated whether the individual tendency to attribute intentionality towards robots modulates the degree of inclusion of the iCub robot during the Cyberball game. Results suggested that the individuals' stance towards collectivism and tendency to attribute a mind to robots both predicted the level of social inclusion of the $\mathrm{iCub}$ robot in our version of the Cyberball game.
\end{abstract}

Keywords: Human-Robot Interaction, Cyberball, Collectivism, Mind attribution.

*These authors equally contributed to this work. 


\section{Introduction}

Recent literature showed that culture leads to cognitive and perceptual differences. For instance, individuals belonging to the Western culture are more analytical and oriented towards independence, while those belonging to East European cultures are more holistic and prone to interdependency [1]. Notably, these differences can also affect the phenomenon of social inclusion, which can substantially vary depending on the context [2]. For instance, affiliation is crucial in collectivistic cultures, where individuals strive for harmony and avoidance of conflicts. Thus, they tend to focus more on positive aspects of social interactions [3]. In contrast, the core of individualistic cultures is selfreliance, leading people to benefit less from the experience of being included by others, relative to people from collectivistic cultures [4]. In this context, little is known about the potential role of culture in social inclusion of robots. Recent studies in HumanRobot Interaction (HRI) demonstrated that individuals' behaviors towards robots might vary across different cultures [5]. For instance, when comparing people from Eastern (China, Japan) and Western countries (Germany), participants expressed different degrees of likeability, satisfaction, trust, and engagement towards robots [6]. Interestingly, the cultural background also affects the distance kept with robots during social interactions [7]; even facial expression recognition has been demonstrated to be culturally dependent [8]. In this context, individualism-collectivism is one of the main dimensions of culture, used as a means to explain how people represent themselves in relation to others [9]. Recent findings in HRI have shown that belonging to an individualistic rather than a collectivistic culture can influence individuals' attitudes towards robots during an interaction. For example, people from collectivistic societies prefer an implicit communication style in the robot, whereas people from individualistic societies prefer an explicit and straightforward, communication style [10]. Interestingly, the cultural background resulted to be particularly relevant also for anthropomorphizing, and mind attribution towards robots [11-13]. For example, recent findings pointed out that people tend to "deny" mind attribution to robots that are categorized as members of the out-group, based on certain features such as skin color [11] or facial morphology [12]. Nevertheless, to the best of our knowledge, no previous studies investigated whether cultural differences modulate people's tendency towards social inclusion of robots, as a function of mind perception and attribution of intentionality.

\section{$1.1 \quad$ Aim}

The present study had two aims. First, we were interested in evaluating whether cultural differences modulated individuals' tendency to socially include robots as members of their own in-group. To this purpose, we tested two samples of UK and Chinese participants, who were chosen as representative of an individualistic, Western culture and of a collectivistic, East Asian culture, respectively [13].

Notably, individual cultural values (cultural stance) might not be in line with the cultural orientation at the national level. Therefore, we administered the Cultural Values 
Scale (CVS), a 26-items dimensional scale that measures cultural stance at the individual level [14], with a particular focus on the Collectivism subscale. This subscale evaluates to what extent a person displays a collectivistic orientation, defined as being sensitive to in-group influences, loyal to in-group norms, and prone to harmony [14]. In order to measure participants' individual tendency towards social inclusion of robots, we developed a modified version of the Cyberball game [15, 16], a well-established paradigm to investigate social ostracism and social exclusion [17]. In our version, participants were instructed to play a ball-tossing game with two other players, represented by avatars of another human and the humanoid robot iCub [18]. During the game, participants were asked to choose which player they wanted to throw the ball to, being as fast as possible. Notably, both the human player and iCub were programmed to alternate between the participant and the other player, with equal probability of throwing the ball to either of them. Given these premises, we hypothesized that cultural differences, both at a national and individual level, would predict the willingness to pass the ball to the robot (that is to include the robot as an in-group member). More specifically, we hypothesized that collectivist culture (at the national and/or individual level) would mean more social inclusion of the robot.

Our second aim was to investigate whether attribution of intentionality towards robots modulates the cultural differences in tendency to socially include the robot in the Cyberball game. To this purpose, we decided to administer the Waytz questionnaire [19], a 7-items subscale of the Anthropomorphism questionnaire adapted from [20], which measures to what extent people ascribe to robots characteristics that are inherently human, such as intentions, desires, and free will. In other words, the more people would attribute intentionality to robots, the more they would ascribe human-like characteristics to them, thereby considering robots closer to human beings. According to this reasoning, cultural differences would be predictive of the willingness to perceive the robot as a social partner, as a function of attribution of intentionality. Thus, we hypothesized that participants with collectivistic cultural stance would be more likely to ascribe intentionality to robots, and also to pass the ball more often to the robot.

\section{$2 \quad$ Materials and Methods}

\subsection{Sample}

120 participants were recruited to take part in the study. Data were collected through the online platform Prolific (https://www.prolific.co/). As inclusion criteria, we selected the following: age range (18-45 years old), fluent English to ensure that participants understood the instructions of the experiment, handedness (right-handed), and nationality. Specifically, half of participants were English $\left(\mathrm{M}_{\text {age }}=25.5 ; \mathrm{SD}_{\text {age }}=5\right.$, males $=15$, Other $=2)$, whereas the other half were Chinese $\left(\mathrm{M}_{\text {age }}=26.3 ; \mathrm{SD}_{\text {age }}=4.5\right.$, males $=22$ ). Additionally, information about participants' educational levels was collected (see Table 1). The study was approved by the local Ethical Committee (Comitato Etico Regione Liguria) and was conducted in accordance with the Code of Ethics of the 
World Medical Association (Declaration of Helsinki, 2013). All participants gave informed consent by ticking an appropriate box in the online form and were naïve to the purpose of the experiment. They all received an honorarium of $4.40 £$ for their participation.

\begin{tabular}{|c|c|c|c|c|}
\hline & \multicolumn{3}{|c|}{$\begin{array}{c}\text { Educational } \\
\text { Levels }\end{array}$} \\
\hline Sample & Bachelor & Master & Ph.D. & NA \\
\hline English & $20(33.7 \%)$ & $9(15.2 \%)$ & $3(5 \%)$ & $27(45.8 \%)$ \\
\hline Chinese & $17(28.8 \%)$ & $28(47.5 \%)$ & $7(11.9 \%)$ & $8(13.6 \%)$ \\
\hline
\end{tabular}

Table 1. Educational levels declared by participants before starting the experiment.

\subsection{Procedure}

As pre-task questionnaires, participants were asked to fill out the Waytz questionnaire, [19], and the Cultural Values Scale (CVS) [14]. Afterward, participants were given instructions to perform the Cyberball game [15-17] (see Fig.1). Before starting the game, a short presentation of the two players was given to participants, who were introduced to both the human confederate ("This is Davide") and the iCub robot ("This is iCub"). The human confederate was depicted as a Caucasian young male, in a neutral background as well as the iCub robot. We did not manipulate its gender, ethnicity, or race, as previous findings [21] showed that the presence of humans does not affect individuals' tendency to attribute human traits to robots. However, further studies should deeply investigate whether these aspects have an impact on the probability of robot choice, which was beyond the scope of this paper.

Each trial started with the presentation of both the human player and $\mathrm{iCub}$, on the left and the right side of the screen, respectively. The name of the participant ("You") was displayed at the bottom. The act of tossing the ball was simulated by presenting a onesecond animation of a ball. When participants received the ball, they were invited to wait until their identification (i.e. "You") turned from black into red before passing the ball. Then, they had $500 \mathrm{~ms}$ to decide which player to pass the ball to. Specifically, to choose the player on their left side (Human) they had to press the " $\mathrm{D}$ " key, whereas the "K" key was to choose the player on the right side of the screen (Robot). To make sure that participants' responses were not biased by the different locations of the keys, before the experiment we asked participants to use a standard QWERTY keyboard to perform the task. If participants took more than $500 \mathrm{~ms}$ to give their response, a red "time-out" statement was displayed in the middle of the screen and the trial was rejected as invalid. The task comprised 100 trials in which participants received the ball (plus trials to replace timeouts). 

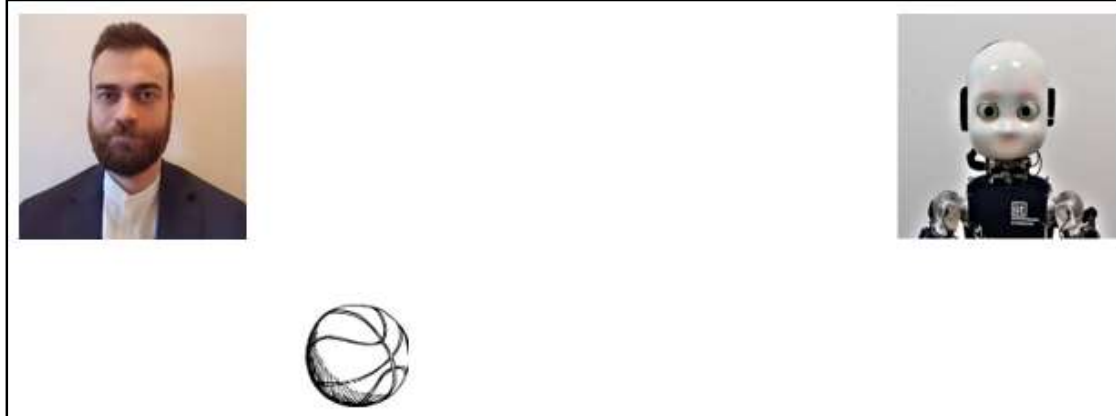

You

Fig.1. Schematic representation of the Cyberball game.

At the end of the Cyberball game, participants filled out a modified version of the Overlap of Self, Ingroup, and Outgroup (OSIO) scale [22], comprising four items that visually represent the closeness between the two players of the Cyberball (i.e., the human player and the iCub robot, see Fig.2). From top to bottom, the picture of the two players tended to get closer. Participants were asked to choose the picture that, according to them, most precisely represented the current closeness between the human player and iCub. For all four items, we assigned a value of 1 to the first and a value of 4 to the last picture. Thus, higher score indicated more closeness between the two players.

All questionnaires and the Cyberball game (stimuli presentation, response timing), and data collection were programmed by using Psychopy v.2020.1.3 [23].

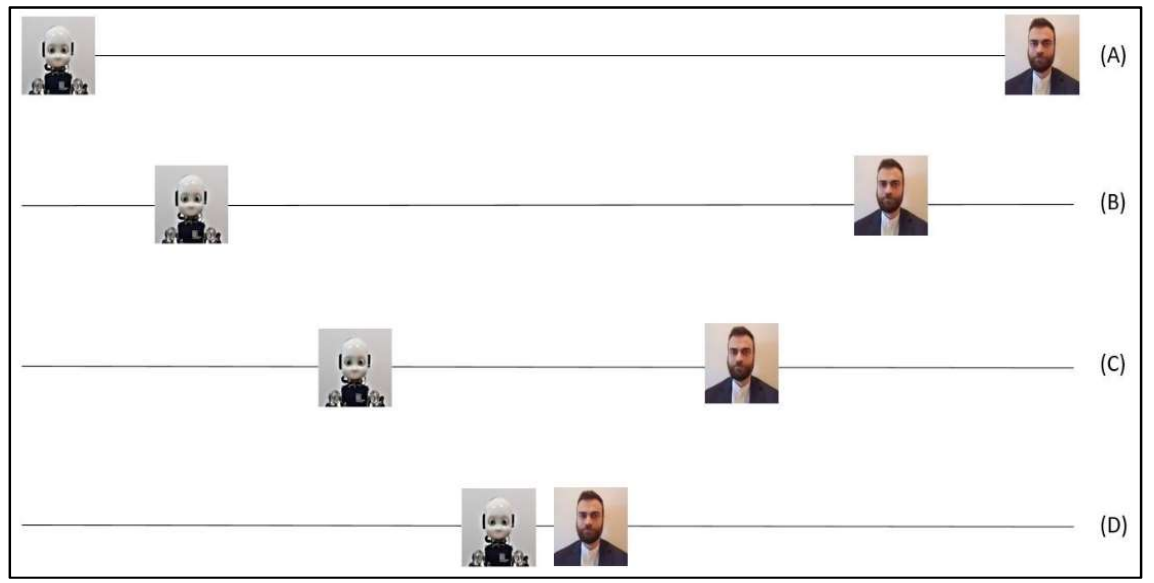

Fig.2. Schematic representation of the modified version of the OSIO scale. 


\section{Results}

\subsection{Cyberball: data pre-processing}

All data were pre-processed with R v.4.0.2 [24], and JASP v.0.14.1 (2020). Data of one participant from the English sample were not saved, and therefore they were not included in the analyses. Data of participants with less than $70 \%$ of valid trials (valid trials meant pressing either " $\mathrm{D}$ " or " $\mathrm{K}$ " within $500 \mathrm{~ms}$ after the signal to throw the ball, that is after the "You" word became red) were discarded from all pre-processing procedures and subsequent analyses, resulting in a final sample of $\mathrm{N}=115$ (UK, $\mathrm{N}=57$; Chinese $\mathrm{N}=58$ ). Moreover, data were cleaned based on participants' reaction times (RTs): RTs that were faster than $100 \mathrm{~ms}$ were discarded as they were considered anticipatory responses $(43.19 \%$ of the trials). Finally, we checked for outliers, excluding trials that were \pm 2 SD from each participants' mean RTs [25] (5.52\% of the trials were excluded). For each presented effect, we will report between square brackets the following statistics: unstandardized coefficient of regression (b), standard error (SE), zstatistics (or t-statistics where appropriate), p-value, and 95\% confidence interval (95\% C.I.) (or $\mathrm{R}^{2}$ where appropriate).

\subsection{The effect of cultural differences on social inclusion of the robot.}

To test whether cultural differences modulate individuals' tendency to socially include robots as in-group members, frequency of robot choice was analyzed with a logistic regression model, with nationality (Chinese/English) as a fixed factor and score calculated by the Collectivism subscale of the CVS questionnaire [14] as a covariate. Results showed a main effect of Collectivism $[b=0.15, \mathrm{SE}=0.03, \mathrm{z}=4.12, \mathrm{p}=<0.001, \mathrm{CI}=$ $(0.08 ; 0.22)]$, but no interaction with nationality was observed. Specifically, the more people displayed a collectivistic orientation, the more frequently they tended to pass the ball to the robot (see Fig.3).

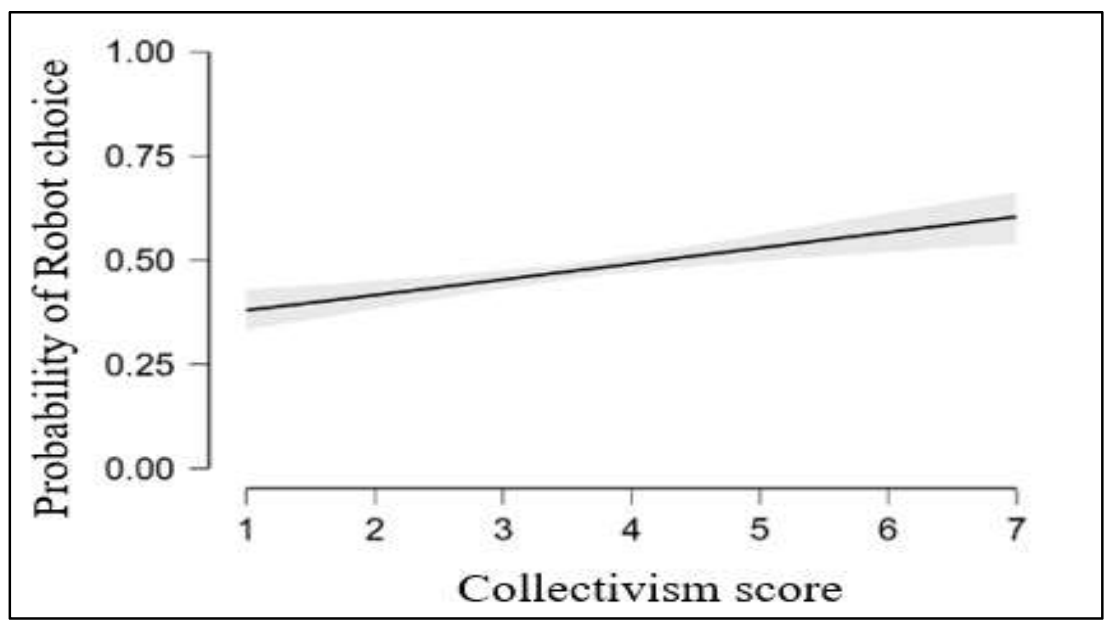


Fig.3. Logistic regression model showing the relationship between the probability of choosing the robot and the Collectivism score.

\subsection{The effect of intentionality attribution on social inclusion of the robot.}

To test whether participants' nationality predicts social inclusion of robot as a function of intentionality attribution, frequency of robot choice was analyzed with a logistic regression model, with nationality (Chinese/English) as a fixed factor and Waytz score as a covariate. Results showed a significant two-way interaction between nationality and Waytz score $[\mathrm{b}=0.1, \mathrm{SE}=1.1, \mathrm{z}=1.99, \mathrm{p}=0.04, \mathrm{CI}=(0.001 ; 0.192)]$. To further investigate this interaction, we performed two logistic regression models, separately for each nationality (Chinese/English). For Chinese participants, results showed that the more they tended to attribute intentionality to robots, the less frequently they passed the ball to the robot $[b=-0.09, S E=0.04, z=-2.49, p=0.01, C I=(-0.16 ;-0.02)]$. Notably, English participants did not show this pattern $[\mathrm{b}=0.006, \mathrm{SE}=0.03, \mathrm{z}=0.14, \mathrm{p}=0.88$, $\mathrm{CI}=(-0.05 ; 0.07)]$ (see Fig.4).

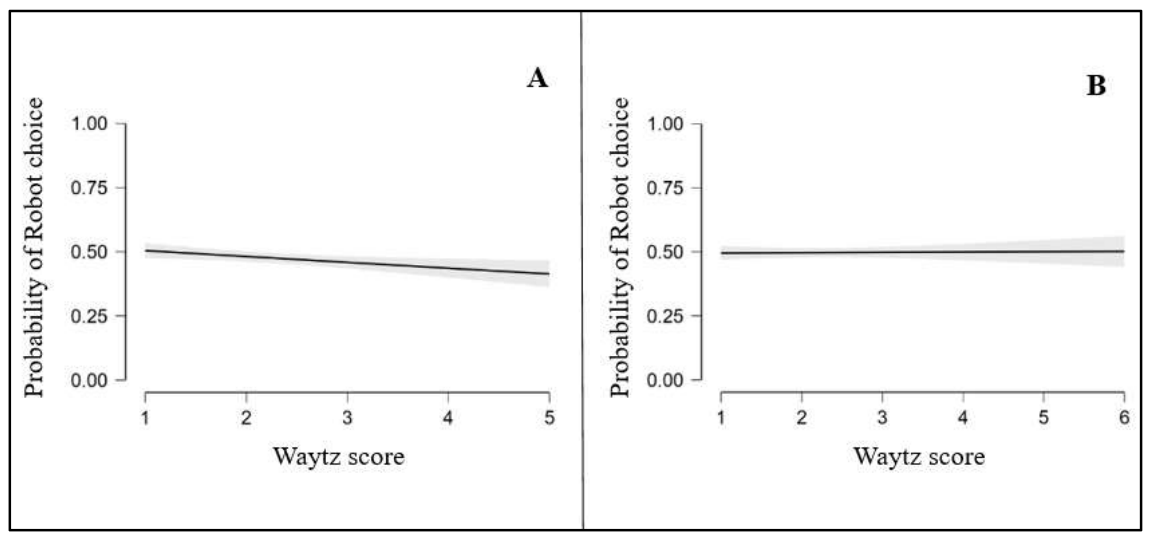

Fig.4. Logistic regression model, showing the relationship between the probability of choosing the robot and the Waytz score, separately according to nationality (Panel A: Chinese participants; Panel B: English participants).

\subsection{OSIO results}

To check for participants' perceived level of closeness as a function of nationality, we performed a linear regression considering the OSIO scale as the dependent variable and nationality as the independent variable. The main effect of nationality emerged as significant $\left[\mathrm{b}=0.46, \mathrm{SE}=0.11, \mathrm{t}=3.86, \mathrm{p}=0.0001, \mathrm{R}^{2}=0.06, \mathrm{CI}=(0.1 ; 0.77)\right]$, showing that Chinese participants scored lower at the OSIO scale compared to the UK participants, thus reporting a lower level of perceived closeness between the human and the robot agent. 


\section{Discussion}

The present experiment aimed at investigating whether cultural differences, operationalized as nationality of participants (Chinese/English) would predict the social inclusion of the robot as a function of (i) individual collectivistic stance and (ii) attribution of intentionality towards robots. The tendency to consider the robot as a social in-group partner was operationalized as the probability of including the humanoid iCub robot in a ball-tossing game, namely the Cyberball [15-17]. With respect to the first aim, results showed that the more participants displayed a collectivistic stance, the more they tended to pass the ball to the robot, regardless of their nationality (Chinese/English). As a consequence, what seems to matter for social inclusion of robots is not national identity but individuals' cultural stance. With respect to the second aim (ii), results showed that, for Chinese participants, the more they tended to attribute intentionality to robots, the less they chose to pass the ball to iCub in the Cyberball game. This was not the case for the UK participants, among whom the individual tendency to attribute intentionality towards robots did not relate to the likelihood of socially including iCub.

This intriguing pattern can perhaps be explained as follows: in collectivist cultures the more an individual is perceived as autonomous, and having a "mind of one's own" (phrases used in the Waytz questionnaire), the less the individual is perceived as an ingroup member. "Autonomy" or "mind of one's own" might be perceived as being against the collectivist values. On the other hand, in individualistic cultures, being autonomous or "having a mind of one's own" might be still more compatible with ingroup membership, and hence no negative relationship between Waytz score and social inclusion has been found for the UK participants. However, results from OSIO scale seem to be in contrast with our hypothesis, as they showed that Chinese participants perceived less "closeness" to the robotic agent. Therefore, this speculative interpretation of the patterns of results needs to be further examined in future research.

At present, our preliminary findings could potentially contribute to design robots that can take into account people's cultural stance, at both individual and social levels. For example, the degree of "autonomy" and "intentionality" displayed by the robot should be tailored to individuals' cultural background, as it could bias the perception of the robot as a social partner.

\section{Conclusions}

Taken together, our results suggest that social inclusion of robots is influenced by the individual collectivistic stance. Moreover, attribution of intentionality towards robots impacts the social exclusion of the robotic agent, but only among members of a collectivist culture. Future research should investigate (and replicate) whether these findings generalize to other nationalities and cultures and also to ecological settings with an embodied humanoid robot. 


\section{Funding}

This study has received support from the European Research Council under the European Union's Horizon 2020 research and innovation program, ERC Starting grant ERC2016-StG-715058, awarded to Agnieszka Wykowska. The content of this paper is the sole responsibility of the authors. The European Commission or its services cannot be held responsible for any use that may be made of the information it contains.

\section{References}

1. Varnum, M. E. W., Grossmann, I., Kitayama, S., \& Nisbett, R. E. (2010). The origin of cultural differences in cognition: The social orientation hypothesis. Current Directions in Psychological Science, 19(1), 9-13. https://doi.org/10.1177/0963721409359301

2. Pfundmair, M., Aydin, N., Du, H., Yeung, S., Frey, D., \& Graupmann, V. (2015). Exclude Me If You Can: Cultural Effects on the Outcomes of Social Exclusion. Journal of Cross-Cultural Psychology, 46(4), 579-596. https://doi.org/10.1177/0022022115571203

3. Takahashi, K., Ohara, N., Antonucci, T. C., \& Akiyama, H. (2002). Commonalities and differences in close relationships among the Americans and Japanese: A comparison by the individualism/collectivism concept. International Journal of Behavioral Development, 26(5), 453-465. https://doi.org/10.1080/01650250143000418

4. Pfundmair, M., Graupmann, V., Du, H., Frey, D., \& Aydin, N. (2015). Suddenly included: Cultural differences in experiencing re-inclusion. International Journal of Psychology, 50(2), 85-92. https://doi.org/10.1002/ijop.12082

5. Lim, V., Rooksby, M., \& Cross, E. S. (2020). Social Robots on a Global Stage: Establishing a Role for Culture During Human-Robot Interaction. International Journal of Social Robotics. https://doi.org/10.1007/s12369-02000710-4

6. Rau, P. L. P., Li, Y., \& Li, D. (2010). A cross-cultural study: Effect of robot appearance and task. International Journal of Social Robotics, 2(2), 175-186. https://doi.org/10.1007/s12369-010-0056-9

7. Remland, M. S., Jones, T. S., \& Brinkman, H. (1991). Proxemic and haptic behavior in three European countries. Journal of Nonverbal Behavior, 15(4), 215-232. https://doi.org/10.1007/BF00986923

8. Jack, R. E., Blais, C., Scheepers, C., Schyns, P. G., \& Caldara, R. (2009). Cultural Confusions Show that Facial Expressions Are Not Universal. Current Biology, 19(18), 1543-1548. https://doi.org/10.1016/j.cub.2009.07.051

9. Markus, H. R., \& Kitayama, S. (1991). Culture and the self: Implications for cognition, emotion, and motivation. Psychological review, 98(2), 224.

10. Papadopoulos, I., \& Koulouglioti, C. (2018). The Influence of Culture on Attitudes Towards Humanoid and Animal-like Robots: An Integrative Review. 
Journal of Nursing Scholarship, 50(6), 653-665. https://doi.org/10.1111/jnu.12422

11. Eyssel, F., \& Loughnan, S. (2013). "It Don't Matter If You're Black or White"?, 422-431. https://doi.org/10.1007/978-3-319-02675-6_42

12. Krumhuber, E. G., Swiderska, A., Tsankova, E., Kamble, S. V., \& Kappas, A. (2015). Real or artificial? Intergroup biases in mind perception in a crosscultural perspective. PLoS ONE, 10(9), 1-14. https://doi.org/10.1371/journal.pone.0137840

13. Hofstede, G. (2011). Dimensionalizing Cultures: The Hofstede Model in Context. Online Readings in Psychology and Culture, 2(1), 1-26. https://doi.org/10.9707/2307-0919.1014

14. Yoo, B., Donthu, N., \& Lenartowicz, T. (2011). Measuring hofstede's five dimensions of cultural values at the individual level: Development and validation of CVSCALE. Journal of International Consumer Marketing, 23(34), 193-210. https://doi.org/10.1080/08961530.2011.578059

15. Williams, K. D., Cheung, C. K., \& Choi, W. (2000). Cyberostracism: effects of being ignored over the Internet. Journal of personality and social psychology, 79(5), 748 .

16. Williams, K. D., \& Jarvis, B. (2006). Cyberball: A program for use in research on interpersonal ostracism and acceptance. Behavior research methods, 38(1), 174-180.

17. Bossi, F., Gallucci, M., \& Ricciardelli, P. (2018). How social exclusion modulates social information processing: A behavioural dissociation between facial expressions and gaze direction. PLoS ONE, 13(4), 1-25. https://doi.org/10.1371/journal.pone.0195100

18. Metta, G., Natale, L., Nori, F., Sandini, G., Vernon, D., Fadiga, L., ... Montesano, L. (2010). The iCub humanoid robot: An open-systems platform for research in cognitive development. Neural Networks, 23(8-9), 1125-1134. https://doi.org/10.1016/j.neunet.2010.08.010

19. Ruijten, P. A. M., Haans, A., Ham, J., \& Midden, C. J. H. (2019). Perceived Human-Likeness of Social Robots: Testing the Rasch Model as a Method for Measuring Anthropomorphism. International Journal of Social Robotics, 11(3), 477-494. https://doi.org/10.1007/s12369-019-00516-z

20. Waytz, A., Morewedge, C. K., Epley, N., Monteleone, G., Gao, J. H., \& Cacioppo, J. T. (2010). Making sense by making sentient: effectance motivation increases anthropomorphism. Journal of personality and social psychology, 99(3), 410-435. https://doi.org/10.1037/a0020240

21. Marchesi, S., Ghiglino, D., Ciardo, F., Perez-Osorio, J., Baykara, E., \& Wykowska, A. (2019). Do we adopt the intentional stance toward humanoid robots? Frontiers in psychology, 10, 450. https://doi.org/10.3389/fpsyg.2019.00450

22. Schubert, T. W., \& Otten, S. (2002). Overlap of Self, Ingroup, and Outgroup: Pictorial Measures of Self-Categorization. Self and Identity, 1(4), 353-376. https://doi.org/10.1080/152988602760328012

23. Peirce, J., Gray, J. R., Simpson, S., MacAskill, M., Höchenberger, R., Sogo, H., ... Lindeløv, J. K. (2019). PsychoPy2: Experiments in behavior made easy. 
Behavior Research Methods, 51(1), 195-203. https://doi.org/10.3758/s13428018-01193-y

24. Bunn, A., \& Korpela, M. (2013). A language and environment for statistical computing. Foundation for Statistical Computing, 2, 1-12.

25. Harald Baayen, R., \& Milin, P. (2010). Analyzing reaction times. International Journal of Psychological Research, 3(2), 12-28. https://doi.org/10.21500/20112084.807 